\title{
Cannibalism as a life boat mechanism
}

\author{
F. van den Bosch ${ }^{1}$, A. M. de Roos', and W. Gabriel ${ }^{2}$ \\ 1 Institute of Theoretical Biology, State University of Leiden, Groenhovenstraat 5 , \\ 2311 BT Leiden, The Netherlands \\ ${ }^{2}$ Max-Planck-Institut für Limnologie, Abt. für Ökophysiologie, Postfach 165 , \\ D-2320 Plön, Federal Republic of Germany
}

\begin{abstract}
Under certain conditions a cannibalistic population can survive when food for the adults is too scarce to support a non-cannibalistic population. Cannibalism can have this lifeboat effect if (i) the juveniles feed on a resource inaccessible to the adults; and (ii) the adults are cannibalistic and thus incorporate indirectly the inaccessible resource. Using a simple model we conclude that the mechanism works when, at low population densities, the average yield, in terms of new offspring, due to the energy provided by one cannibalized juvenile is larger than one.
\end{abstract}

Key words: Structured population model - McKendrick equation - Subcritical bifurcation - Solver

\section{Introduction}

Populations of predators are sometimes observed to live for a considerable period of time with very little or no prey available. For example, Johnson and Walker (1973), reported a population of the predatory cyclopoid copepod Cyclops strenuus abyssorum in Loch Leven, which lived for three successive years with extremely low levels of zooplankton available as food for the adults. They argued that the amount of prey present was far too little to sustain a population. Cannibalism, regularly observed in live and preserved samples, was considered to be an important factor. Similarly, Collette et al. (1977) state that in some perch populations juveniles represent the major food source for the adults. According to Nikolskii (1969) cannibalism often enables perch to live in lakes with no other prey available for the adults. Popova and Sytina (1976) report that in some lakes in the USSR, the fish fauna is composed only of perch (Perca fluviatilis).

In his review on intraspecific predation Polis (1981) lists an additional three examples all of which concern fish species. He also indicates a mechanism that allows these populations to survive by means of cannibalism. Firstly, the juveniles have to feed on a resource inaccessible to the adults. Secondly, the adults are cannibalistic and thus incorporate indirectly the inaccessible resource. Using this 
extra energy, an adult can increase its reproductive rate or decrease its death rate. Of course, cannibalism also increases the juvenile death rate.

The mechanism which we refer to as the "life boat mechanism", can work in species whose juveniles use substantially different food from the adults, such as is the case in scorpions, waterbugs, squid, newts, frogs, some copepod species and several fish species (Polis 1981).

Of course the examples about populations sustaining on cannibalism are extreme cases where one is inclined to search for possible causes. The life boat mechanism can, however, also be important in situations with a prey population present but not productive enough to support a non-cannibalistic population.

Gabriel (1985a, b, c) was the first to study this life boat mechanism in a theoretical context. He showed that cannibalism can indeed decrease the risk of extinction. Due to the complexity of the simulation model used, no simple rule could be obtained about the conditions under which cannibalism allows the population to persist. An analytical approach may facilitate the derivation of such a rule.

Hence, in this paper we use a simple model, to answer the question: Under what conditions can a cannibalistic population survive when food for the adults is too scarce to support a non-cannibalistic population?

\section{What to look for?}

A central quantity throughout this paper will be the net reproduction, $R_{0}$, which we define as the expected number of female offspring produced by one female throughout her life at infinitesimally low population density where density dependent factors, including cannibalism, can be neglected.

Note. It should be pointed out that, in the ecological literature " $R_{0}$ " and the term "net-reproduction" are sometimes used also for the density dependent case. Other sources do restrict the interpretation of $R_{0}$ to the density independent situation and use $R_{n}$, or some equivalent notation, for the density dependent situation. We prefer to restrict the use of $R_{0}$, where the 0 refers to zero (read infinitesimally low) population density, to the density independent situation only.

In models for the dynamics of a population it is commonly assumed that the per capita birth rate decreases and/or the per capita death rate increases, for at least some age classes, with increasing population density. In such situations it can usually be shown that when $R_{0}<1$ only the trivial steady state exists, while a branch of non-trivial steady states bifurcates supercritically from $R_{0}=1$. The trivial steady state is stable for $R_{0}<1$ and unstable for $R_{0}>1$. When a species is cannibalistic increasing juvenile population density results in a larger birth rate and/or a smaller death rate for the adults. Cushing (1985) showed that it is possible, in such situations, that the branch of non-trivial steady states bifurcates subcritically from $R_{0}=1$. In some situations the branch bends back again to values of $R_{0}>1$.

Given that there exists a non-trivial steady state for $R_{0}<1$, there might be some population trajectories that remain bounded away from zero for all time. Then a cannibalistic population can survive when a non-cannibalistic population goes extinct. Finding such situations is the subject of the next sections. 


\section{The model}

Symbols that are frequently used in the subsequent description of the model are summarized in Table 1.

Assume that the amount of food eaten by a juvenile of a certain age is constant through time. This assumption allows us to write down an age structured model for the juveniles because there is a fixed relation between age, $a$, and energy content, $E(a)$. Furthermore, assume that a juvenile becomes an adult at a fixed age, $\bar{a}$. Let $n(t, a)$ denote the age distribution of the juveniles. The time evolution of this distribution is governed by a McKendrick type equation,

$$
\frac{\partial n}{\partial t}+\frac{\partial n}{\partial a}=-[\mu+\tilde{C}(a) A(t)] n(t, a) ; \quad a \leqslant \bar{a}
$$

where $\mu$ is the per capita natural death rate, which is assumed to be age independent, $A(t)$ is the total number of adults and $\tilde{C}(a)$ is the attack rate of an adult on juveniles of age $a$. We assume a linear functional response. We choose to write $\tilde{C}$ as the product

$$
\tilde{C}(a)=C h(a)
$$

where $C$ is the total (per adult) cannibalistic pressure an individual experiences during its juvenile period and $h(a)$ is the relative vulnerability to cannibalism of juveniles of age $a$, with support $\left[a_{\min }, a_{\max }\right] \subset(0, \bar{a})$ and

$$
\int_{0}^{\bar{a}} h(a) d a=1 .
$$

Assume that the adults do not differ in their population dynamical behaviour. Their dynamics can then be described by an ordinary differential equation

$$
\frac{d A}{d t}=n(t, \bar{a})-f(I(t)) A(t)
$$

Table 1. List of important symbols

\begin{tabular}{ll}
\hline Symbol & Explanation \\
\hline$a$ & Age \\
$\bar{a}$ & Age at which an individual matures \\
$A(t)$ & Total number of adults \\
$\tilde{C}(a)$ & Attack rate per adult on juveniles of age $a$ \\
$C$ & Total cannibalistic pressure (per adult) which an individual experiences during its \\
$E(a)$ & juvenile period \\
$f(I(t))$ & Energy content of an individual of age $a$ \\
$h(a)$ & Per capita adult death rate \\
$I(t)$ & Relative vulnerability to cannibalism at age $a$ \\
$J(t)$ & Total rate of energy intake per adult \\
$n(t, a)$ & Rate of energy intake per adult through cannibalism \\
$R_{0}$ & Age distribution of the juveniles \\
$\mu$ & Net reproduction \\
$Z$ & Per capita juvenile death rate \\
$\zeta$ & Rate of energy intake per adult from an extraneous food source \\
\hline
\end{tabular}


where $n(t, \bar{a})$ is the influx of adults which is equal to the out-flow (maturation) of juveniles at age $\tilde{a}, f(I)$ is the per capita death rate, which is assumed to be a function of the total rate of energy intake, $I$. We assume that $f(I)<\infty ; f^{\prime}(I)<0$ and $\lim _{I \rightarrow \infty} f(I)>0$. The biology behind these assumptions is that "well fed" individuals are less vulnerable to infectious diseases, can escape predators more efficiently, etc.

The total rate of energy intake of an adult, $I$, is the sum of the energy intake from an extraneous food source, $Z$, and the energy intake through cannibalism, $J(t)$. The intake from the extraneous source is assumed to be constant.

$$
I(t)=Z+J(t) .
$$

The rate of energy intake per adult through cannibalism is given by

$$
J(t)=\int_{0}^{\bar{a}} \tilde{C}(a) E(a) n(t, a) d a
$$

Finally, the per capita birth rate is assumed to depend linearly on the total energy intake, i.e. a linear numerical response. The population birth rate, therefore, takes the form

$$
n(t, 0)=\zeta(Z+J(t)) A(t)
$$

where $\zeta$ is the conversion efficiency; i.e. $\zeta^{-1}$ is the amount of energy needed to produce one newborn. A biological restriction on $\zeta$ is

$$
\zeta E(0)<1 .
$$

Equation (1e) concludes our model specification. In the following sections we analyze the behaviour of the model, with particular attention to the steadystates. This will be done using the net-reproduction as a bifurcation parameter. Expressed in mechanistic parameters:

$$
\begin{aligned}
R_{0} & =\int_{0}^{\infty}\left(\begin{array}{c}
\text { probability to } \\
\text { survive till } \\
\text { age } a
\end{array}\right) \cdot\left(\begin{array}{c}
\text { rate of offspring } \\
\text { production at age } a \\
\text { at } I(t)=Z
\end{array}\right) d a \\
& =\zeta Z \frac{\exp (-\mu \bar{a})}{f(Z)} .
\end{aligned}
$$

\section{The steady states}

We shall only consider steady states of $A(t)$, denoted by $\hat{A}$. Steady state values of all other quantities can be expressed as monotonically increasing functions of $\hat{A}$. Among the steady states is the trivial steady state

$$
\hat{A}=\mathbf{0} \text {. }
$$

All non-trivial steady states are found from

$$
F(\hat{A}, Z):=\beta(\hat{A}) \gamma(\hat{A}, Z) \rho(\hat{A}, Z)=1
$$


with $\beta(\hat{A})=\exp (-\mu \bar{a}-C \hat{A})$, the probability to survive until maturity; $\gamma(\hat{A}, Z)=$ $1 / f(Z+J(\hat{A}))$, the expected longevity of an adult; $\rho(\hat{A}, Z)=\zeta(Z+J(\hat{A}))$, the number of newborns produced per unit time per adult; and

$$
J(\hat{A})=\frac{Z \Phi(\hat{A})}{1-\Phi(\hat{A})}
$$

with

$$
\Phi(\hat{A})=\zeta \hat{A} C \int_{0}^{\bar{a}} h(\alpha) E(\alpha) \exp \left(-\mu \alpha-C \int_{0}^{\alpha} h(\sigma) d \sigma \hat{A}\right) d \alpha .
$$

Equation (4) is readily interpreted as the condition that every individual replaces itself on average.

It can be shown that there is only one continuous branch of non-trivial steady states; no isolated branches of non-trivial steady states exist (Appendix 1).

Now first note that when $\hat{A} \downarrow 0, F(\hat{A}, Z) \rightarrow R_{0}$. So, the branch of steady states indeed bifurcates from $R_{0}=1$. The first property to be considered is concerned with the direction of bifurcation. The branch of non-trivial steady states bifurcates sub-(super-)critically if

$$
\left.\frac{d \hat{A}}{d R_{0}}\right|_{R_{0}=1}<(>) 0
$$

Using the implicit function theorem, this derivative can be calculated from

$$
\frac{d \hat{A}}{d R_{0}}=\frac{d \hat{A}}{d Z} \frac{d Z}{d R_{0}}=-\left(\frac{\partial F}{\partial \hat{A}}\right)^{-1}\left(\frac{\partial F}{\partial Z}\right)\left(\frac{d R_{0}}{d Z}\right)^{-1} .
$$

Lengthy but straightforward calculations show that the condition for subcritical bifurcation is

$$
\zeta\left(1-Z \frac{f^{\prime}(Z)}{f(Z)}\right) \psi>1
$$

where

$$
\psi=\int_{0}^{\bar{a}} h(\sigma) E(\sigma) e^{-\mu \sigma} d \sigma .
$$

The quantity $\psi$ can be interpreted as the average energy gain from cannibalizing one juvenile at infinitesimally low adult density. The cannibal converts this energy into (i) additional reproduction; and (ii) an increased lifespan during which it converts an additional amount of extraneous food into newborns. The number of newborns produced directly from $\psi$ is equal to the product of $\psi$ and the efficiency factor $\zeta$. The average contribution to the energy intake rate due to cannibalizing one juvenile at low population density, equals the quotient of $\psi$ and the cannibals average lifespan at low population density, $1 / f(Z)$. This contribution feeds through to extending the cannibals lifespan. The increase in lifespan per unit of additional energy intake is, at low population density:

$$
\frac{d}{d Z} \frac{1}{f(Z)}=-\frac{f^{\prime}(Z)}{f^{2}(Z)} .
$$


Dividing this by $1 / f(Z)$ gives the average amount added to the lifespan of an adult by cannibalizing one juvenile. Multiplication of the additional time lived with the reproductive output from the extraneous food source, $\zeta Z$, finally gives the total number of newborns during the increase in lifespan. Therefore, we have a biological interpretation for all terms of the criterion in (5).

In conclusion we can say that the branch of non-trivial steady states bifurcates subcritically from the trivial steady state if the average yield, in terms of new juveniles, from cannibalizing one juvenile at low population density is larger than one. We will refer to (5) as the "mean yield criterion".

A second property of the branch is concerned with its direction when $\hat{A} \uparrow \infty$. From (4) it is seen that when $\hat{A} \uparrow \infty, \beta(\hat{A}) \downarrow 0$. Because $f$ is always larger than zero and bounded, $\gamma(\hat{A})$ is always larger than zero and bounded. In order to satisfy (4). when $\hat{A} \uparrow \infty$, it is necessary that $\rho(\hat{A}) \rightarrow+\infty$. Furthermore, note that for $\hat{A} \uparrow \infty$

$$
\hat{A C h}(a) \exp \left(-\hat{A} C \int_{0}^{a} h(\sigma) d \sigma\right) \rightarrow \delta\left(a-a_{\min }\right)
$$

where $a_{\text {min }}$ is the minimum of the support of $h(a)$. This leads to

$$
\lim _{\hat{A} \uparrow \infty} \rho(\hat{A}, Z)=\zeta Z \frac{1}{1-\zeta E\left(a_{\min }\right) \exp \left(-\mu a_{\min }\right)} .
$$

From which it can be concluded that when $\hat{A} \uparrow \infty$,

$$
Z \rightarrow(-) \infty \quad \Leftrightarrow \quad R_{0} \rightarrow(-) \infty \quad \text { if } \Omega>(<) 1
$$

where

$$
\Omega=\zeta E\left(a_{\min }\right) \exp \left(-\mu a_{\min }\right)
$$

When the number of adults becomes infinite the fraction of (the infinite number of) juveniles that fall victim to cannibalism will be cannibalized at age $a_{\min } . \Omega$ can be interpreted as the number of newborns produced directly from the energy provided by one juvenile of age $a_{\min }$; i.e. $\Omega$ is the minimal yield. It is obvious that when $\Omega$ is larger than one the population can (when started with the appropriate initial conditions) grow out of bound. When $\Omega$ is smaller than one, the population can never grow out of bound regardless of the increased lifespan of an adult due to cannibalism.

Proof by contradiction. Assume that the population grows out of bound. Then, the energy intake per adult through cannibalism becomes infinite (see (1d)). When $\Omega$ is smaller than one, the loss in number of individuals for the population due to the cannibalistic activity of adults becomes infinite. The finite increase in the adult longevity, and through this the finite increase in reproduction from the energy provided by the extraneous resource, can never compensate for this infinite loss. Thus, the population cannot grow out of bound.

These two properties lead to four possible shapes of the branch of non-trivial steady states (Fig. 1). There might also exist branches with several bends, turning back and forth several times. We have been unable to elucidate the conditions under which such branches exist in the general model. 


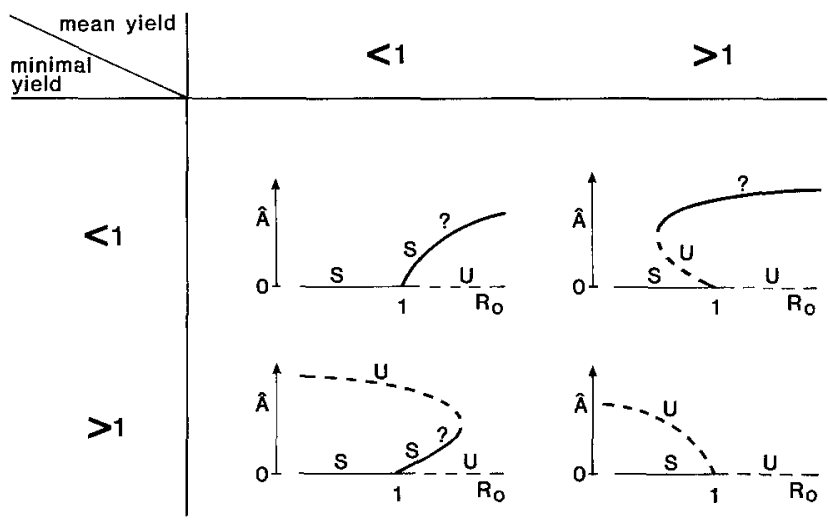

Fig. 1. The four simplest shapes of branches of non-trivial steady states and their expected stability properties ( $\mathrm{s}=$ stable; $\mathrm{u}=$ unstable; $?=$ unknown). Horizontal: $R_{0}$ is the net-reproduction. Vertical: $\hat{A}$ is the steady state adult density. When the mean yield, in terms of new juveniles, from cannibalizing one juvenile at infinitesimally low population density is smaller (larger) than unity; $d \hat{A} /\left.d R_{0}\right|_{\hat{A}=0}<$ $(>) 0$. When the yield from cannibalizing one individual at the minimal vulnerable age is smaller (larger) than unity; $\lim _{\hat{A} \rightarrow \infty} R_{0}=(-) \infty$

\section{Some remarks about stability}

Linearization about the trivial steady state leads to the characteristic equation

$$
F(\lambda)=0
$$

where

$$
F(\lambda)=-1+R_{0} \exp (-\lambda \bar{a})-\frac{\lambda}{f(Z)}
$$

from which we can conclude that the trivial steady state is stable if $R_{0}<1$ and unstable when $R_{0}>1$.

The characteristic equation associated with the non-trivial steady state turns out to be extremely unwieldy. An analysis, if possible in general at all, is outside the scope of this paper. Owing to the lack of continuity of the age specific death rate, model (1), unfortunately, does not fit into the framework of the general theory developed by Cushing (1985). The principle of exchange of stability, however, suggests that the non-trivial steady state is stable near the bifurcation point in the case of supercritical bifurcation. At larger values of $R_{0}$ the steady state might lose stability, through a Hopf bifurcation, and give rise to a stable limit cycle and possibly other dynamics at even larger values of $R_{0}$. In the case of subcritical bifurcation a steady state is expected to be unstable when $d \hat{A} / d R_{0}<$ 0 . At least near the bifurcation point the unstable steady state is expected to be a saddle. When in this situation the branch turns back there is a turning point where $d \hat{A} / d R_{0}=\infty$. Continuity arguments can be used to conclude that if the turning point is close to the bifurcation point, the upper part of the branch must consist of stable equilibria at least close to the turning point. In general, however, nothing can be said about the stability of the upper part. When the minimal yield is larger than one the biological interpretation leads one to expect that the part of the branch where $d \hat{A} / d R_{0}<0$ consists of saddles. All this is summarized in Fig. 1. 

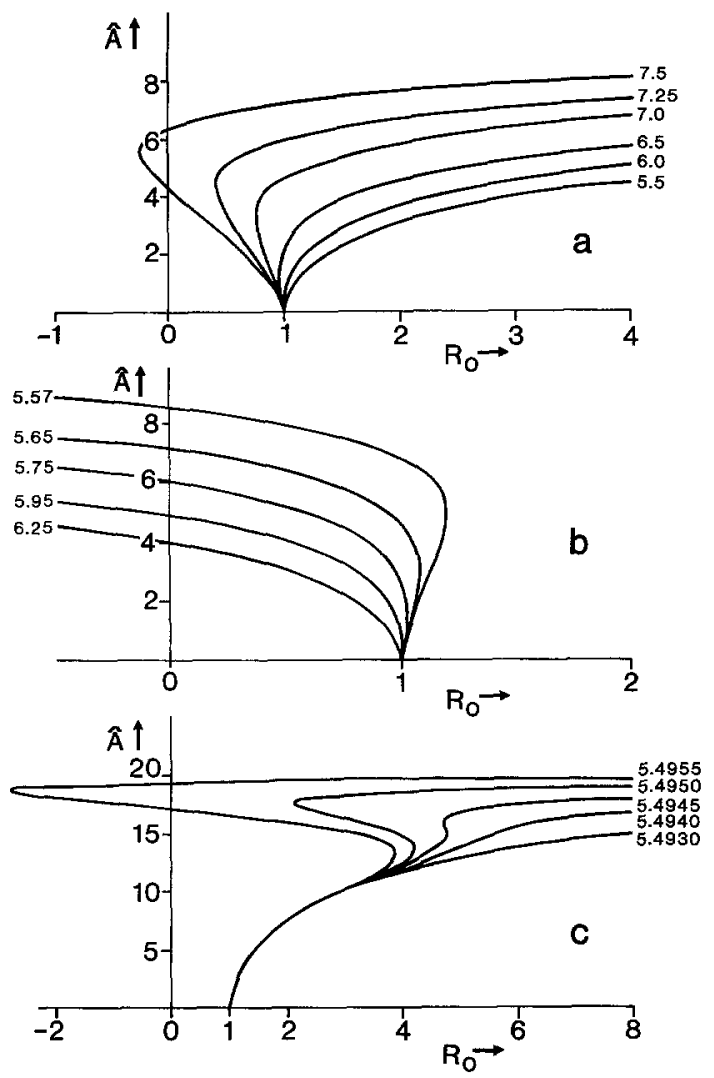

Fig. 2a-c. Branches of non-trivial steady states of the special case investigated in Sect. 6. Horizontal: $R_{0}$ is the net-reproduction. Vertical: $\hat{A}$ is the steady state adult density. In all cases the widths of the vulnerable age window, $w$, is 0.6 and the death rate of the juveniles equals 2.0 . a Branches for the middle of the vulnerability window, $m$ is 0.4 and various values of the efficiency factor times the growth rate of the juveniles, $\zeta \alpha$; b branches for $m=0.7$ and various value of $\zeta \alpha$; $\mathbf{c}$ branches for $m=0.7$ and various values of $\zeta \alpha$

\section{A numerical example}

Although much insight is gained from the analysis in the preceding sections, some questions still remain. These questions concern the existence of branches with several bends, the boundedness of the population trajectories, and the lowest possible $R_{0}$ for which the population may survive in the case of subcritical bifurcation. In this section a simple special case is used to illustrate the theory and to get some insight in the above questions.

For simplicity, we assume that the adult per capita death rate is independent of the energy uptake:

$$
f(Z+J(t))=f
$$

This allows us to rewrite the steady state equation (4) as

$$
R_{0}=\frac{1-\zeta C \int_{0}^{\bar{a}} h(a) E(a) \exp \left(-\mu a-C \int_{0}^{a} h(\sigma) d \sigma \hat{A}\right) d a \hat{A}}{\exp (-C \hat{A})}
$$




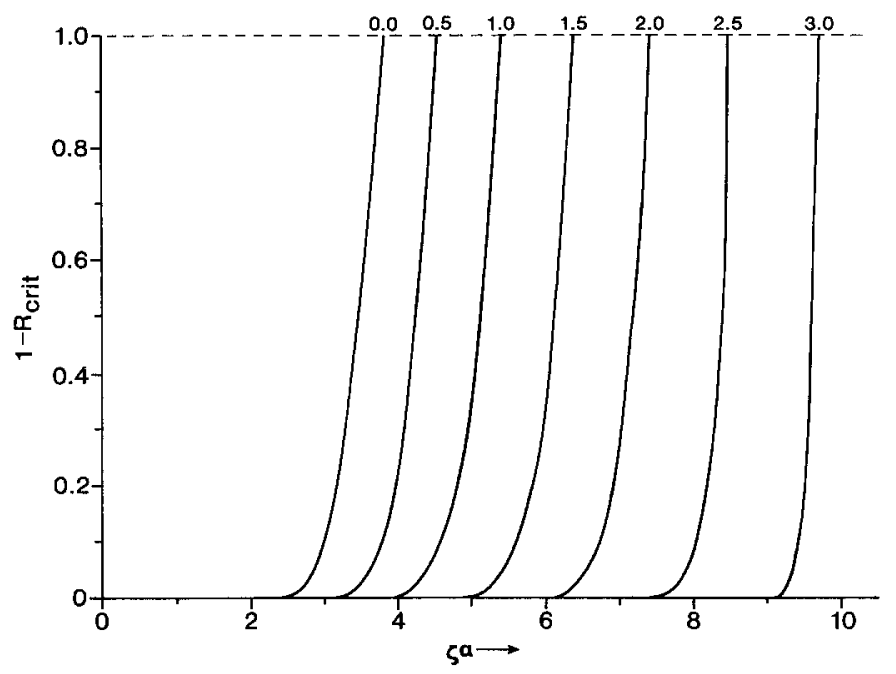

Fig. 3. The quantity $1-R_{\text {crit }}$ as a function of the product of the efficiency factor and the juvenile growth rate, $\zeta \alpha$; for various values of the juvenile death rate, $\mu$. The quantity $1-R_{\text {crit }}$ indicates how severe a food deprivation the population can cope with. In all cases the widths and middle of the vulnerable age window is 0.6 and 0.4 , respectively

Furthermore, the energy content is assumed to increase linearly with age,

$$
E(a)=\alpha a,
$$

and the juveniles in the age window $\left[m-\frac{1}{2} w ; m+\frac{1}{2} w\right]$ are all equally vulnerable to cannibalism where $m$ is the middle and $w$ is the widths of the window, and invulnerable outside this window. For this special case it is also possible to rewrite the model as a system of six delay differential equations, (see Appendix 2) which facilitates studying its dynamical behaviour numerically.

As we see from Eq. (8), $C$ can be absorbed into $\hat{A}$. Furthermore, $\bar{a}$ is a scaling factor of the time. Without loss of generality we can put $\bar{a}=1$.

Steady states. Figure 2a illustrates the change from super- to subcritical bifurcation in the case where the minimal yield is smaller than one. In some situations a non-trivial steady state exists for $R_{0}=0$, i.e. with no extraneous food available. This might explain the extreme cases discussed in the Introduction. For this type of branch it is relevant to know how small $R_{0}$ can become before the population goes extinct. Denote by $R_{\text {crit }}$ the value of $R_{0}$ at the turning point of the branch. The population can cope with any food deprivation smaller than the quantity $1-R_{\text {crit }}$. Figure 3 depicts this quantity as a function of $\zeta \alpha$ for various values of $\mu$. We see that small changes in the parameter values can have a large effect on $1-R_{\text {crit }}$. This figure gives the impression that if the mean yield of one cannibalized juvenile is only slightly larger than one, the population can survive periods of severe food deprivation. 


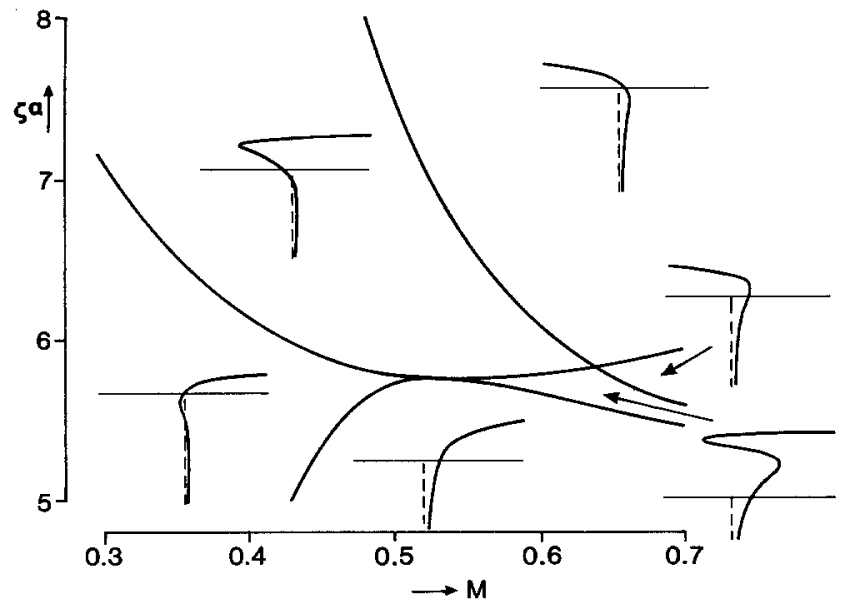

Fig. 4. Subdivision of the $\zeta \alpha-m$ (i.e. product of the efficiency factor and juvenile growth rate middle of the vulnerable age window) parameter space into areas where the various shapes of branches of non-trivial steady states occur. The widths of the vulnerable age window is 0.6 and the juvenile death rate is 2.0. To ease the interpretation of how various branches develop from each other a branch is drawn in each area; also values for $\hat{A}<0$ are depicted

Figure $2 \mathrm{~b}$ illustrates the change from super- to subcritical bifurcation when the minimal yield of a cannibalized individual is larger than one.

In this special case branches with two bends exist (Fig. 2c). Situations with more bends have not been found. We do not yet have a clear biological interpretation for the existence of these double bended branches.

Figure 4 gives an overview of the $(\zeta \alpha, m)$-parameter space with the regions where the different types of branches occur. In all cases investigated, the region with double bended branches is rather small. Also note that these branches bend back far below $R_{0}=1$, while the criterion for subcritical bifurcation is not yet fulfilled.

Population trajectories and stability. To simplify the discussion we define a "larger" ("smaller") steady state as one having a larger (smaller) steady state value for $\hat{A}$ than the one under consideration. Furthermore, starting a simulation "below" ("above") some steady state means that we take a value of $\hat{A}$ slightly smaller (larger) than the steady state value of $\hat{A}$, fill this value into the equations for the steady states of all other variables and use the values calculated to start the simulation. The stability properties of the steady states closely agree with the expected properties discussed in the previous section. Parts of branches for which $d \hat{A} / d R_{0}<0$, always consists of unstable steady states showing saddle like behaviour. If, in such a situation, a simulation is started below the steady state, the population trajectories converge to (i) the lower steady state if it is stable (be it the trivial one or some non-trivial one) or (ii) to the periodic solution around the lower steady state. Starting a simulation above the steady state, the population either grows out of bound, when there is no larger steady state, or converges to the larger steady state if it is stable and to the periodic solution around it otherwise. 
When $d \hat{A} / d R_{0}>0$ the steady state is either stable or unstable. If it is unstable the population trajectories converge to a stable limit cycle. Simulations up to $R_{0}=10$ did not show other types of dynamics. All steady states were found to be stable when the complete juvenile period is vulnerable. When there is a stable limit cycle for $R_{0} \leqslant 1$, the lowest values of the variables always exceeded one third of these steady state values.

\section{Concluding remarks}

We have shown that under certain conditions a cannibalistic population can survive when food for the adults is too scarce to support a non-cannibalistic population. This phenomenon is based on the capability of the adults to incorporate indirectly a resource which is only accessible to the juveniles. Using the analytical results of Sect. 4 and generalizing from the numerical example we may, with some reservations, conclude that a cannibalistic population can survive periods of food deprivation if, at low population densities, the average yield in terms of new juveniles produced from the energy provided by one cannibalized individual is larger than one.

Increasing juvenile population density increases the total reproductive output of an adult. In a more general sense this phenomenon is known in ecology as the Allee-effect (Allee 1938). In this light the life boat mechanism can be interpreted as a somewhat unusual kind of Allee-effect.

In order to keep the model tractable many simplifying assumptions were made. Some of them can, however, be relaxed without changing the mean yield criterion. Assume, for instance, that the death rate of the juveniles is age dependent. This only changes the expression (and not the interpretation) of the probability to survive till age $\bar{a}$ in (5).

A more realistic satiating functional response, $R(Z, n)$, would take the form

$$
R(Z, n)=C^{*} h(a) \theta(Z, n)
$$

where $\theta$ is the correction factor for density dependent effects. For a two prey Holling type II (Murdoch and Oaten (1975)) age structured functional response

$$
\theta(Z, n)=\frac{1}{1+\varepsilon_{1} Z+C^{*} \int_{0}^{\bar{a}} h(a) n(t, a) \varepsilon_{2}(a) d a}
$$

Where $\varepsilon_{1}$ is the handling time of the extraneous food source and $\varepsilon_{2}(a)$ is the handling time of a juvenile of age $a$. At infinitesimally low population densities, at which the mean yield criterion is derived, Eq. (9) simplifies to

$$
R(Z, n)=C^{*} h(a) \theta(Z, 0) .
$$

For the Holling functional response,

$$
\theta(Z, 0)=\frac{1}{1+\beta_{1} Z}
$$

Redefining $C:=C^{*} \theta(Z, 0), C$ can be interpreted as the total cannibalistic pressure a juvenile experiences at extraneous food level $Z$. The total cannibalistic pressure does not appear in the mean yield criterion. 
For a more general numerical response the per capita reproduction rate of an adult, denoted by $P$, takes the form

$$
P(Z, J)=g(Z+J(t)),
$$

where $g$ is the rate of offspring production as a function of the energy intake. At low population densities this becomes

$$
P(Z, J)=g^{\prime}(Z)\left[\frac{g(Z)}{g^{\prime}(Z)}+J(t)\right],
$$

where $g^{\prime}(Z)$ can be interpreted as the efficiency factor, $\zeta$, at a given extraneous food level $Z$. $g / g^{\prime}$ represents the effective rate of extraneous food intake, which is simply a redefinition of $Z$. So, a more general numerical response will also not change the mean yield-criterion.

In the derivation of the model, we assumed that the energy intake rate of a juvenile is constant. It is more realistic, however, to assume that the food source for the juveniles is a dynamic variable itself. We then have to write down a size structured model where $E$ is a function of both age and time, and $h$ is a function of size. However, at infinitesimally low population densities this food source is at its carrying capacity and the size of a juvenile becomes a fixed function of age.

Of course the shape of the branch of non-trivial steady states, including its direction when $\hat{A} \uparrow \infty$, can be influenced by the above modifications. Since our main purpose here was to investigate when cannibalism can sustain a population afloat through periods of adult food shortage (which turned out to be determined by the mean yield criterion) further elaboration on this point is beyond the scope of this paper. In conclusion we expect the biological interpretation of the mean yield-criterion to be fairly independent of the exact model specifications. Therefore, the presented lifeboat mechanism and the mean-yield criterion is of some general use and may motivate more theoretical and experimental work on the subject of cannibalism.

Acknowledgements. We are grateful to the organizers of "the first Texel workshop on the modelling of physiologically structured populations" for bringing us together and to the Max Planck Institut für Limnologie for hospitality and financial support during a visit. Furthermore, we thank all participants of the "cannibalism lunches" at the Institute of Theoretical Biology for fruitful discussions. We thank L. J. Weider, S. S. Kilham and M. A. Mort for improving the English and Reinhard Bürger for comments on an early draft of the paper. We are also grateful to Y. M. Zitman-de Graaf for typing the manuscript and to G. P. G. Hock for drawing the figures.

\section{Appendix 1: The branch of non-trivial steady states}

In this appendix we show that there is only one continuous branch of non-trivial steady states for $\hat{A}>0$.

In equilibrium the following holds:

$$
\left\{\begin{array}{l}
\hat{n}(a)=\hat{n}(0) \exp \left(-\mu a-C \int_{0}^{a} h(\alpha) d \alpha \hat{A}\right) \\
\hat{A}=\frac{\hat{n}(\bar{a})}{f(Z+J)}, \quad \hat{J}=C \int_{0}^{\bar{a}} h(\alpha) E(\alpha) \hat{n}(\alpha) d \alpha .
\end{array}\right.
$$


From these equations we conclude, using the assumption $0<f(I)<\infty, \forall I$, that

$$
\hat{A}>0 \Leftrightarrow \hat{n}(a)>0 \Leftrightarrow \hat{n}(0)>0 \Leftrightarrow \hat{J}>0
$$

and

$$
\hat{A}<\infty \Leftrightarrow \hat{n}(a)<\infty \Leftrightarrow \hat{n}(0)<\infty \Leftrightarrow \hat{J}<\infty .
$$

Now we note the following:

(i) In Sect. 4 we showed that any branch of non-trivial steady states bifurcated at $R_{0}=1$ from the trivial steady state $\hat{A}=0$.

(ii) Since $\hat{J}=[Z \Phi(\hat{A}) / 1-\Phi(\hat{A})]$ and $0<\Phi(\hat{A})<\infty$ for $0<\hat{A}<\infty$, the condition $0<\hat{J}<\infty$ implies that for a fixed value $\hat{A}=\hat{A}^{*}$, all the corresponding values $Z=Z^{*}$, which together constitute solution pairs $\left(\hat{A}^{*}, Z^{*}\right)$ of Eq. (4) are strictly positive if $1-\Phi\left(\hat{A}^{*}\right)>0$ and strictly negative if $1-\Phi\left(\hat{A}^{*}\right)<0$.

(iii) From Eq. (4) we find

$$
\begin{aligned}
\frac{\partial F}{\partial Z} & =\beta(\hat{A})\left\{\gamma(\hat{A}, Z) \frac{\partial \rho}{\partial Z}+\rho(\hat{A}, Z) \frac{\partial \gamma}{\partial Z}\right\} \\
& =\beta(\hat{A})\left\{\gamma(\hat{A}, Z)-\rho(\hat{A}, Z) \frac{f^{\prime}}{\zeta f^{2}}\right\} \frac{\zeta}{1-\Phi(\hat{A})} .
\end{aligned}
$$

Our assumptions concerning $f$ require that $\gamma(\hat{A}, Z)>0$ and $f^{\prime}<0$. Moreover, since solutions of Eq. (4) only exist for $Z$ and $1-\Phi(\hat{A})$ both positive or both negative, $\rho(\hat{A}, Z)=\zeta(Z+\hat{J})=$ $[\zeta Z / 1-\Phi(\hat{A})]>0$. In these cases $F(\hat{A}, Z)$ is increasing monotonically if $1-\Phi(\hat{A})>0$ and $Z>0$ and decreasing monotonically if $1-\Phi(\hat{A})<0$ and $Z<0$. Together with (ii) this leads to the conclusion that for $0<\hat{A}<\infty$ there is at most one value of $Z$, such that $(\hat{A}, Z)$ is a solution of Eq. (4).

(iv) If $\hat{A}<\infty$, then

$$
\beta(\hat{A})>0 \quad \text { and } \quad \gamma(\hat{A}, Z)>0 .
$$

The latter inequality always holds, due to our assumptions of $f$. If $Z$ and $1-\Phi(\hat{A})$ are both positive or both negative, then

$$
\|Z\| \rightarrow \infty \Leftrightarrow \rho(\hat{A}, Z) \rightarrow \infty
$$

and therefore Eq. (4) has no solution for $Z \rightarrow \pm \infty$ provided $\hat{A}<\infty$. Since $Z$ is directly linked to $R_{0}$, the same holds for $R_{0} \rightarrow \pm \infty$ and $\hat{A}<\infty$. In Sect. 4 we concluded that

$$
\hat{A} \uparrow \infty \Leftrightarrow R_{0} \rightarrow \pm \infty
$$

from which we conclude that the only solution for $R_{0} \rightarrow \pm \infty$ is $\hat{A} \rightarrow \infty$.

Together (i)-(iv) imply that there exists exactly one branch of non-trivial steady states, which bifurcates at $R_{0}=1$ from the trivial steady state $\hat{A}=0$, while for $\hat{A} \uparrow \infty, R_{0} \rightarrow \pm \infty$, dependent upon the value of $\Omega$ in Eq. (6b).

\section{Appendix 2: Model equations of the numerical example}

In the special case of Sect. 6 the population can be divided into four age classes. Define $S$ (mall), $V$ (ulnerable), $L$ (arge), and $A$ (dults) to be the numbers of individuals in the age interval $\left(0, a_{1}\right)$; $\left(a_{1}, a_{2}\right) ;\left(a_{2}, \bar{a}\right)$ and $(\bar{a}, \infty)$, respectively where $a_{1}=m-\frac{1}{2} w$ and $a_{2}=m+\frac{1}{2} w$. Following Gurney et al. (1983), define $R_{i}(t)$ to be the recruitment into age class $i(i=S, V, L, A)$ from the preceding age class and $b(t)$ to be the birth rate. The differential equations for the numbers of individuals in the various age classes are (writing $C^{*}$ for $C /\left(a_{2}-a_{1}\right)$ )

$$
\begin{aligned}
& \frac{d S}{d t}=b(t)-R_{V}(t)-\mu S(t) \\
& \frac{d V}{d t}=R_{V}(t)-R_{L}(t)-C^{*} V(t) A(t)-\mu V(t) \\
& \frac{d L}{d t}=R_{L}(t)-R_{A}(t)-\mu L(t) \\
& \frac{d A}{d t}=R_{A}(t)-f A(t)
\end{aligned}
$$


with

$$
\begin{aligned}
b(t) & =\zeta(Z+J(t)) A(t) \\
R_{V}(t) & =b\left(t-a_{1}\right) \exp \left(-\mu a_{1}\right) \\
R_{L}(t) & =R_{V}\left(t-a_{2}+a_{1}\right) P_{V}(t) \\
R_{A}(t) & =R_{L}\left(t-\bar{a}+a_{2}\right) \exp \left(-\mu\left(\bar{a}-a_{2}\right)\right)
\end{aligned}
$$

where $P_{V}(t)$ is the probability to survive the vulnerable period. This probability can be calculated from

$$
P_{V}(t)=\exp \left\{-\int_{t-a_{2}+a_{1}}^{t}\left[\mu+C^{*} A(\sigma)\right] d \sigma\right\} .
$$

Differentiation with respect to time yields a differential equation for $P_{V}(t)$

$$
\frac{d P_{V}}{d t}=P_{V}(t) C^{*}\left\{A\left(t-a_{2}+a_{1}\right)-A(t)\right\} .
$$

As a last step we define a differential equation for $J(t)$. Differentiating Eq. (1d) with respect to time yields

$$
\frac{d J}{d t}=\alpha C^{*} \int_{a_{1}}^{a_{2}} a \frac{\partial n}{\partial t} d a
$$

and substitution of Eq. (1a) gives

$$
\begin{aligned}
\frac{d J}{d t} & =\alpha C^{*} \int_{a_{1}}^{a_{2}} a\left(-\frac{\partial n}{\partial a}-\mu n-C^{*} n A\right) d a \\
& =\alpha C^{*}\left\{-a_{2} R_{V}(t)+a_{1} R_{S}(t)+V(t)\right\}-\left(\mu+C^{*} A(t)\right) J(t)
\end{aligned}
$$

completing the system of equations.

For this system of equations numerical solutions can be obtained using the program template SOLVER (Maas et al. (1984)). Differentiating the integral equation for $P_{V}(t)$ has one drawback; it introduces an eigenvalue zero into the system. Discretization can subsequently induce numerical instability. A variety of cases is known in which the solutions of such possibly numerically unstable systems are accurate as long as small integration time steps are used (Nisbet, personal communication). For this reason numerical solutions were often recalculated with a smaller time step. Furthermore, some of the numerical solutions were checked using a very general method for the numerical integration of the type of partial differential equations occurring in structured population models (de Roos 1988).

\section{References}

1. Allee, W. C.: Cooperation among animals. New York: Henry Schuman 1938

2. Collette, B. B., Ali, M. A., Hokanson, K. E. F., Nagiec, M., Smirnov, S. A., Thorpe, J. E., Weatherley, A. H., Willemsen, J.: Biology of percids. J. Fish. Res. Board Can. 34, 1890-1899 (1977)

3. Cushing, J. M.: Equilibria in structured populations. J. Math. Biol. 23, 15-39 (1985)

4. De Roos, A. M.: Numerical methods for structured population models: the escalator boxcar train. Numerical methods for partial differential equations 4(3): 173-195 (1988)

5. Gabriel, W.: Can cannibalism be advantageous in cyclopoids? A mathematical model. Verh. Internat. Verein. Limnol. 22, 3164-3168 (1985a)

6. Gabriel, W.: Overcoming food limitations by cannibalism: A model study on cyclopoids. Arch. Hydrobiol. Beih. 21, 373-381 (1985b)

7. Gabriel, W.: Simulation komplexer Populationsdynamik. In: Möller, D. P. F. (ed.) Simulationstechnik (Informatik Fachberichte, vol. 109, pp. 318-324) 1985c

8. Gurney, W. S. C., Nisbet, R. M., Lawton, J. H.: The systematic formulation of tractable singlespecies population models incorporating age structure. J. Anim. Ecol. 52, 479-495 (1983). 
9. Johnson, D., Walker, A. F.: The zooplankton of Loch Leven. Kinross. Proc. R.S. Edinb. (B) 74, 285-294 (1972/73)

10. Maas, P., Gurney, W. S. C., Nisbet, R. M.: SOLVER - An adaptable program template for initial value problem solving. Applied Physics Industrial Consultants. Glasgow: Univ. of Strathclyde 1982; revised 1984

11. Murdoch, W. W., Oaten, A.: Predation and population stability. Advances in ecological research 9, 1-131 (1975)

12. Nikolskii, G. V.: Theory of fish population dynamics. Edinburgh: Oliver \& Boyd 1969

13. Polis, G. A.: The evolution and dynamics of intraspecific predation. Ann. Rev. Ecol. Syst. 12, 225-251 (1981)

14. Popova, O. A., Sytina, L. A.: Food and feeding relations of eurasian perch (Perca fluviatilis) and pikeperch (Stizostedio luciperca) in various waters of the USSR. J. Fish. Res. Board Can. 34, 1559-1570 (1977)

Received April 7/Revised July 25, 1988 